\title{
Analysis of the pion wave function in light-cone formalism*
}

\author{
Tao Huang, Bo-Qiang Ma and Qi-Xing Shen \\ Center of Theoretical Physics, China Center of Advanced Science \\ and Technology (world Laboratory) Beijing, China \\ and \\ Institute of High Energy Physics, Academia Sinica \\ P.O.Box 918(4), Beijing 100039, China $^{\dagger}$
}

\begin{abstract}
Several general constraints are suggested to analyze the pionic valence-state wave function. It is found that the present model wave functions used in lightcone formalism of perturbative quantum chromodynamics have failed these requirements to fit the pionic formfactor data and the reasonable valence-state structure function which does not exceed the pionic structure function data for $x \rightarrow 1$ simultaneously. Furthermore, it is pointed out that there is a possibility to find model wave functions which can satisfy all of general constraints. Also we show that there are two higher helicity $\left(\lambda_{1}+\lambda_{2}= \pm 1\right)$ components in the light-cone wave function for the pion as a natural consequence from the Melosh rotation and it is speculated that these components should be incorporated into the perturbative quantum chromodynamics.
\end{abstract}

Detailed Version.

To be published in Phys.Rev.D

\footnotetext{
${ }^{*}$ Project supported by National Natural Science foundation of China and the grant LWTZ-1298 of Chinese Academy of Science.

${ }^{\dagger}$ Mailing Address
} 


\section{Introduction}

The hadronic wave functions in terms of quark and gluon degrees of freedom play an important role in the quantum chromodynamics (QCD) predictions for hadronic processes. In the perturbative QCD theory (pQCD) ${ }^{1}$, the hadronic distribution amplitudes and structure functions which enter exclusive and inclusive processes via the factorization theorems ${ }^{2-3}$ at high momentum transfer can be determined by the hadronic wave functions, and therefore they are the underlying links between hadronic phenomena in QCD at large distances (non-perturbative) and small distances (perturbative). If the hadronic wave functions were accurately known, then we could calculate the hadronic distribution amplitudes and structure functions for exclusive and inclusive processes in QCD. Conversely, these processes also can provide phenomenological constraints on the hadronic distribution amplitudes, the hadronic structure functions, and thereby the hadronic wave functions.

Several important non-perturbative tools have been developed which allow specific predictions for the hadronic distribution amplitudes or the hadronic wave functions directly from theory. QCD sum-rule technique ${ }^{4-5}$ and lattice gauge theory ${ }^{6-7}$ provide constraints on the moments of the hadronic distribution amplitude. One thus could model the hadronic distribution amplitudes by fitting the first few moments in terms of Gegenbauer polynomials which are the solutions to the evolution equation of hadronic distribution amplitude ${ }^{2-3}$. The Chernyak-Zhitnitsky (CZ) distribution amplitudes ${ }^{4-5}$ (dubbed wave functions in the original papers) constructed in this way are good in reproducing the correct sign and magnitude as well as scaling behavior of the pion, proton and neutron form factors. However, whether the CZ-like distribution amplitude is the correct pion distribution amplitude is still an open problem and its correctness should not be judged by only its success in reproducing the correct magnitude of the pion form factor. Some earlier lattice Monte Carlo calculations ${ }^{6}$, designed to compute the pion distribution amplitude directly, were unable to distinguish between the asymptotic form and the CZ form. In a recent improved lattice QCD calculation ${ }^{7}$ the second moment of the pion distribution amplitude was found to be small than previous lattice calculations $^{6}$ and the sum-rule calculations ${ }^{4-5}$, and this suggests that the pion distribution amplitude may close to the asymptotic form rather than the $\mathrm{CZ}$ form. From 
another point of view, as different wave functions may give a same distribution amplitude, there are still ambiguities about the wave function even if we know the exact form of the distribution amplitude. Hence it is still necessary to develop methods which specify the hadronic wave functions directly.

In principle, the Bethe-Salpeter formalism ${ }^{8}$ and the more recent discretized lightcone quantization approach ${ }^{9}$ could determine the hadronic wave functions, but in practice there are many difficulties in getting the exact wave functions at present ${ }^{10-11}$. One useful way is to use the approximate bound state solution of a hadron in terms of the quark model as the starting point for modeling the hadronic valence wave function. The Brodsky-Huang-Lepage (BHL) prescription ${ }^{3}$ of the hadronic wave function is in fact obtained in this way by connecting the equal-time wave function in the rest frame and the wave function in the infinite momentum frame, and the corresponding wave function gives a distribution amplitude which is significant different from the CZ-form. In order to give the CZ-like distribution amplitude which is required to fit the experimental data, a phenomenological model for the hadronic valence state wave function has been proposed ${ }^{12-14}$ by adding a factorized function to the BHL wave function. The distribution amplitude is almost the same as the CZ distribution amplitude except for the end-point regions. Recently a light-cone quark model approach of hadrons ${ }^{15}$ has received attention for the reason that the model can simultaneously fit low energy phenomena, the measured high momentum transfer hadron form factor, and the $\mathrm{CZ}$ distribution amplitudes. The hadronic wave function in this approach, as will be shown, is significant different from the factorized wave functions in Refs.12-13 though both of them give the similar CZ-like distribution amplitudes.

The purpose of this paper is to discuss the consequence from physical constraints on the hadronic wave functions, using pion valence state wave function as an example. In Sec.II, we will list several general constraints on the pion valence state wave function ${ }^{3,16}$. We then analyse, in Sec.III, several existing pion wave functions used in light-cone formalism of perturbative QCD. We hope to find a wave function which could give both the approximate $\mathrm{CZ}$ distribution amplitude required to fit the pion form factor data and the reasonable valence state structure function which does not exceed the 
pion structure function data simultaneously, with several constraints on the pion wave functions also satisfied. We find, unfortunately, that the present model wave functions have failed the requirements. Furthermore, the most recent light-cone quark model wave function given in Ref.15 violates the general constraints severely. For example, it gives a probability of finding the valence Fock state in a pion much larger than unity if the correct normalization of the pion distribution amplitude is retained. Hence this wave function suffers from serious flaws. However we show that the power-law form of the pionic wave function can satisfy the requirements and it provides a possible example to find a good wave function to be used in QCD theory. In order to properly evaluate the effect from Melosh rotation ${ }^{17-18}$ connecting the rest frame equal-time wave function and the light-front wave function in the light-cone formalism, we re-construct in Sec.IV the light-cone quark model of the pion in light-front dynamics. We find that the contributions from Melosh rotation in the ordinary helicity $\left(\lambda_{1}+\lambda_{2}=0\right)$ component wave function by using reasonable parameters seem to have no significant effect on the calculated distribution amplitude and structure function in comparison with those from the BHL wave function. However, two higher helicity $\left(\lambda_{1}+\lambda_{2}= \pm 1\right)$ components which were not taken into account in previous perturbative QCD emerge naturally in the full light-cone wave function as a consequence from the Wignier rotation. In Sec.V we present the summary and some comments.

\section{Constraints on the Valence-state Wave Function}

In this paper, we employ the particularly convenient light-cone formalism ${ }^{1-3}$ in which the description of the hadronic wave functions is given by a set of n-particle momentum space amplitude,

$$
\psi_{n}\left(x_{i}, \vec{k}_{\perp i}, \lambda_{i}\right), i=1,2, \ldots n
$$

defined on the free quark and gluon Fock basis at equal "light- cone time" $\tau=t+z$ in physical "light-cone" gauge $A^{+}=A^{0}+A^{3}=0$. Here $x_{i}=k_{i}^{+} / p^{+}$, with $\sum_{i} x_{i}=1$, is the light-cone momentum fraction of quark or gluon $i$ in the n-particle Fock state; $\vec{k}_{\perp i}$, with $\sum_{i} \vec{k}_{\perp i}=0$, is its transverse momentum relative to the total momentum $p^{\mu}$; and $\lambda_{i}$ is its light-cone helicity. Any hadron state can be expanded in terms of this 
complete set of Fock states at equal $\tau$ :

$$
\begin{aligned}
\mid H>=\sum_{n, \lambda_{i}} \int[d x]\left[d^{2} \vec{k}_{\perp}\right] \psi_{n}\left(x_{i}, k_{\perp i}, \lambda_{i}\right) \quad & \prod_{\text {fermions }} \frac{u\left(x_{i} p^{+}, x_{i} \vec{p}_{\perp}+\vec{k}_{\perp i}\right)_{\lambda_{i}}}{\sqrt{x_{i}}} \\
& \prod_{\text {gluons }} \frac{\epsilon\left(x_{i} p^{+}, x_{i} \vec{p}_{\perp}+\vec{k}_{\perp i}\right)_{\lambda_{i}}}{\sqrt{x_{i}}} \mid n>
\end{aligned}
$$

with the normalization condition,

$$
\sum_{n, \lambda} \int[d x]\left[d^{2} \vec{k}_{\perp}\right]\left|\psi_{n}\left(x_{i}, \vec{k}_{\perp_{i}}, \lambda_{i}\right)\right|^{2}=1
$$

where the sum is over all Fock states and helicities, and

$$
\begin{aligned}
{[d x] } & =\delta\left(1-\sum_{i=1}^{n} x_{i}\right) \prod_{i=1}^{n} d x_{i} \\
{\left[d^{2} \vec{k}_{\perp}\right] } & =16 \pi^{3} \delta^{2}\left(\sum_{i=1}^{n} \vec{k}_{\perp i}\right) \prod_{i=1}^{n}\left(d^{2} \vec{k}_{\perp i} / 16 \pi^{3}\right) .
\end{aligned}
$$

The quark and gluon structure functions $G_{q / H}(x, Q)$ and $G_{g / H}(x, Q)$, which control hard inclusive reactions, and the hadron distribution amplitudes $\phi_{H}(x, Q)$, which control hard exclusive reactions, are simply related to these wave functions ${ }^{1-3}$ :

$$
G_{a / H}(x, Q)=\sum_{n} \int\left[d^{2} \vec{k}_{\perp i}\right]\left[d x_{i}\right]\left|\psi_{n}\left(x_{i}, \vec{k}_{\perp i}\right)\right|^{2} \delta\left(x-x_{a}\right), a=q \text { or } g
$$

and

$$
\phi_{H}(x, Q)=\int^{Q^{2}}\left[d^{2} \vec{k}_{\perp i}\right] \psi_{\text {valence }}\left(x_{i}, \vec{k}_{\perp i}\right) .
$$

In the case of inclusive reactions all of the hadron Fock states generally participate; whereas in the case of exclusive reactions perturbative QCD predicts that only the lowest particle number (valence) Fock state contributes to the leading order in $1 / Q$.

In principle the hadronic wave functions determine all properties of hadrons. From the relation between the wave functions and measurable quantities we can get some constraints on the general properties of the hadronic wave functions. In the pionic case two important constraints on the valence state wave function (for the $\lambda_{1}+\lambda_{2}=0$ components) have been derived ${ }^{3}$ from $\pi \rightarrow \mu \nu$ and $\pi^{0} \rightarrow \gamma \gamma$ decay amplitudes:

$$
\int_{0}^{1} d x \int\left(d^{2} \vec{k}_{\perp} / 16 \pi^{3}\right) \psi_{q \bar{q}}\left(x, \vec{k}_{\perp}\right)=f_{\pi} / 2 \sqrt{3}
$$

and

$$
\int_{0}^{1} d x \psi_{q \bar{q}}\left(x, \vec{k}_{\perp}=0\right)=\sqrt{3} / f_{\pi}
$$


where $f_{\pi} \approx 93 \mathrm{MeV}$ is the pion decay constant. The $\lambda_{1}+\lambda_{2} \neq 0$ component valence state wave functions do not contribute in the $\pi \rightarrow \mu \nu$ and $\pi^{0} \rightarrow \gamma \gamma$ processes thereby the presence of the $\lambda_{1}+\lambda_{2} \neq 0$ components does not alter the above two constraints. Experimentally the average quark transverse momentum of the pion, $\left\langle\vec{k}_{\perp}^{2}>_{\pi}\right.$, is of the order $(300 \mathrm{MeV})^{2}$ approximately ${ }^{16}$. The quark transverse momentum of the valence state pion, defined to be

$$
<\vec{k}_{\perp}^{2}>_{q \bar{q}}=\int\left(d^{2} \vec{k}_{\perp} / 16 \pi^{3}\right) d x\left|\vec{k}_{\perp}^{2} \| \psi_{q \bar{q}}\left(x, \vec{k}_{\perp}\right)\right|^{2} / p_{q \bar{q}}
$$

should be large than $\left\langle\vec{k}_{\perp}^{2}\right\rangle_{\pi}$. We thus could require that $\sqrt{\left\langle\vec{k}_{\perp}^{2}\right\rangle_{q \bar{q}}}$ have the value of about a few hundreds $\mathrm{MeV}$, serving as the third constraint. The fourth constraint is the most natural one: The probability of finding the $q \bar{q}$ Fock state in a pion should be not larger than unity,

$$
P_{q \bar{q}}=\int\left(d^{2} \vec{k}_{\perp} / 16 \pi^{3}\right) d x\left|\psi_{q \bar{q}}\left(x, \vec{k}_{\perp}\right)\right|^{2} \leq 1
$$

For the distribution amplitude we compare the calculated one with the CZ-form,

$$
\phi_{C Z}(x)=5 \sqrt{3} f_{\pi} x(1-x)(2 x-1)^{2} .
$$

As to the case of structure function, it should be noticed that the valence state structure function is only one part of the valence structure function of a pion. Hence it is reasonable to require that the calculated valence state structure function not exceed the structure function data for the pion. We will use the NA3 parameterization ${ }^{19}$ of the pion structure function in comparison with the calculated valence state structure function. It should be indicated that the $Q^{2}$ corresponding to the NA3 data ${ }^{19}$ is very large; i.e., $Q^{2}=25(\mathrm{GeV} / \mathrm{c})^{2}$. For $Q^{2}$ of the order of a few $(\mathrm{GeV} / \mathrm{c})^{2}$ the structure function should increase at large $\mathrm{x}$ and decrease at small $\mathrm{x}$ in considering the contributions from QCD $\operatorname{logarithmic~evolution}^{20}$, the higher twist effects and other power-law type sources ${ }^{21}$ for Bjorken scaling violations, with the shape and magnitude not changed too much.

\section{Analysis of Several Existing Wave Functions}

The hadronic wave function depends essentially on the non-perturbative QCD theory, and it exhibits the full complexity of non-perturbative dynamics. It is necessary 
for us to use both theoretical tools and phenomenological constraints in studying the hadronic wave functions. In order to get clear on where assumptions and approximations have been made and where problems may occur, we need to review some previous results in the following analyses of several existing pionic wave functions.

\section{A. The Brodsky-Huang-Lepage Prescription}

Brodsky-Huang-Lepage suggested ${ }^{3}$ a connection between the equal-time wave function in the rest frame and the light-cone wave function by equating the off-shell propagator $\epsilon=M^{2}-\left(\sum_{i=1}^{n} k_{i}\right)^{2}$ in the two frames:

$$
\epsilon=\left\{\begin{array}{l}
M^{2}-\left(\sum_{i=1}^{n} q_{i}^{0}\right)^{2}, \\
M^{2}-\sum_{i=1}^{n}\left[\left(\vec{k}_{\perp i}^{2}+m_{i}^{2}\right) / x_{i}\right], \quad \sum_{i=1}^{n} \vec{k}_{\perp i}^{n}=0, \quad \sum_{i=1}^{n} x_{i}=1 \quad[\text { L.C }]
\end{array}\right.
$$

from which one obtains, for two-particle system with $m_{1}=m_{2}\left(\right.$ i.e., $\left.q_{1}^{0}=q_{2}^{0}\right)$,

$$
\vec{q}^{2} \longleftrightarrow \frac{\vec{k}_{\perp}^{2}+m^{2}}{4 x(1-x)}-m^{2}
$$

Then for two-particle state there is a possible connection between the rest frame wave function $\psi_{C M}(\vec{q})$, which controls binding and hadronic spectroscopy, and the light-cone wave function $\psi_{L C}\left(x, \vec{k}_{\perp}\right)$ by

$$
\psi_{C M}\left(\vec{q}^{2}\right) \longleftrightarrow \psi_{L C}\left(\frac{\vec{k}_{\perp}^{2}+m^{2}}{4 x(1-x)}-m^{2}\right) .
$$

As an example, the wave function of the harmonic oscillator model in the rest frame was obtained from an approximate bound state solution in the quark models for mesons $^{22}$

$$
\psi_{C M}\left(\vec{q}^{2}\right)=A \exp \left(-\vec{q}^{2} / 2 \beta^{2}\right)
$$

By using the connection (3.3) one gets the light-cone wave function,

$$
\begin{aligned}
\psi\left(x_{i}, \vec{k}_{\perp}\right) & =A \exp \left[-\frac{1}{8 \beta^{2}}\left(\frac{\vec{k}_{\perp}^{2}+m^{2}}{x_{1}}+\frac{\vec{k}_{\perp}^{2}+m^{2}}{x_{2}}\right]\right. \\
& =A \exp \left[-\frac{\vec{k}_{\perp}^{2}+m^{2}}{8 \beta^{2} x(1-x)}\right] .
\end{aligned}
$$

The parameters can be adjusted by using the first three constraints, i.e., Eq.(2.6)(2.8), in Sec.II,

$$
m=289 \mathrm{MeV} ; \quad \beta=385 \mathrm{MeV} ; \quad A=0.032 \quad \text { for } \quad\left\langle\vec{k}_{\perp}^{2}>\approx(356 \mathrm{MeV})^{2}\right.
$$


Thus we get the pion distribution amplitude, valence state structure function, and the probability $P_{q \bar{q}}$,

$$
\begin{aligned}
\phi(x) & =\int\left(d^{2} \vec{k}_{\perp} / 16 \pi^{3}\right) \psi\left(x, \vec{k}_{\perp}\right) \\
& =\frac{A \beta^{2}}{2 \pi^{2}} x(1-x) \exp \left[-\frac{m^{2}}{8 \beta^{2} x(1-x)}\right] ; \\
F_{2}^{V}(x) & =\sum_{a=q, \bar{q}} x e_{a}^{2} G_{a / H}(x) \\
& =\frac{5 A^{2} \beta^{2}}{36 \pi^{2}} x^{2}(1-x) \exp \left[-\frac{m^{2}}{4 \beta^{2} x(1-x)}\right] ; \\
P_{q \bar{q}}= & \int\left(d^{2} \vec{k}_{\perp} / 16 \pi^{3}\right) \int d x\left|\psi\left(x, \vec{k}_{\perp}\right)\right|^{2} \\
= & \frac{A^{2} \beta^{2}}{4 \pi^{2}} \int_{0}^{1} x(1-x) \exp \left[-\frac{m^{2}}{4 \beta^{2} x(1-x)}\right] d x \\
\approx & 0.296
\end{aligned}
$$

Fig.1(a) presents the BHL distribution amplitude in comparison with that of the CZ-form and the asymptotic (AS) form ${ }^{3}$,

$$
\phi_{A S}(x)=\sqrt{3} f_{\pi} x(1-x)
$$

which is the leading term of the evolution equation of the pion distribution amplitude for sufficient large $Q^{2}$. It can be seen from Fig.1(a) that the BHL distribution amplitude is very close to that of the AS-form while it is significantly different from that of the CZ-form. By using this distribution amplitude one accounts for only $50 \%$ of the pion form factor data. So it is not the "good" distribution amplitude required to fit the data. However, we see from Fig.1(b), where the NA3 parameterization of the pion structure function and the calculated valence state structure function are presented, that the calculated $F_{2}^{V}(x)$ seems to be a "reasonable" valence state structure function. Therefore the BHL wave function cannot give both a reasonable valence state structure function and a good distribution amplitude simultaneously. The fact that the BHL wave function did not give a "good" distribution amplitude is why the CZ distribution amplitude has received attention since it appeared.

\section{B. The factorized wave functions}

In order to fit the experimental data and to suppress the end- point contributions for the applicability of perturbative QCD, a model for the pion valence wave function has been proposed in Refs.12-13 by simply adding a factorized function $S(x)$ to the BHL wave function, with $S(x)$ specified by

$$
S(x)=\left(x_{1}-x_{2}\right)^{2}=(1-2 x)^{2},
$$


where $x_{1}=x$ and $x_{2}=1-x$. It leads a distribution amplitude

$$
\phi(x)=\frac{A \beta^{2}}{2 \pi^{2}} x(1-x)(2 x-1)^{2} \exp \left[-\frac{m^{2}}{8 \beta^{2} x(1-x)}\right],
$$

which is of the similar shape as that of the CZ-form except for the end-point regions, as shown in Fig.2(a). The parameters are adjusted by the first three constraints,

$$
m=342 \mathrm{MeV} ; \quad \beta=455 \mathrm{MeV} ; A=0.136 \text { for } \quad<\vec{k}_{\perp}^{2}>\approx(343 \mathrm{MeV})^{2}
$$

and the probability $P_{q \bar{q}}$ is 0.364 , which satisfies the fourth constraints, i.e., Eq.(2.9). However, the valence state structure function in this case is

$$
\begin{aligned}
F_{2}^{V}(x) & =\sum_{a=q, \bar{q}} x e_{a}^{2} G_{a / H}(x) \\
& =\frac{5 A^{2} \beta^{2}}{36 \pi^{2}} x^{2}(1-x)(2 x-1)^{2} \exp \left[-\frac{m^{2}}{4 \beta^{2} x(1-x)}\right],
\end{aligned}
$$

which is presented in Fig.2(b). One sees from Fig.2(b) that there is an unreasonablely large hump in the calculated $F_{2}^{V}(x)$. Thereby the factorized wave function (3.11) though is "good" in giving the CZ-like distribution which fits the data well, it is "bad" in giving a reasonable valence state structure function.

One may specify the factorized function $S(x)$ by other possible distribution amplitudes $^{2,3}$ which also are constrained by the first few moments given by sum rules in terms of Gegenbauer polynomials. The distribution amplitudes in Ref.23 do not have the deep $\operatorname{dip}$ at $x=1 / 2$, hence they are different from that of the CZ-form. Fig.3 presents the calculated distribution amplitudes and valence state structure functions by using the factorized wave functions with $S(x)$ specified by ${ }^{23}$

$$
S(x)=1+0.44 C_{2}^{3 / 2}\left(x_{1}-x_{2}\right)+0.25 C_{4}^{3 / 2}\left(x_{1}-x_{2}\right)
$$

and

$$
S(x)=1+(2 / 3) C_{2}^{3 / 2}\left(x_{1}-x_{2}\right)+0.43 C_{4}^{3 / 2}\left(x_{1}-x_{2}\right),
$$

respectively, where $C_{n}^{3 / 2}(\xi)$ is the Gegenbauer polynomials. The parameters are also fixed by the first three constraints in Sec.II. We see from Fig.3(a) that the distribution amplitudes are more broad than the AS-form. However, the calculated valence state structure functions still have unreasonablely large humps, as shown in Fig.3(b). Therefore the factorized wave functions also cannot give both reasonable valence state structure functions and good distribution amplitudes simultaneously. 


\section{The light-cone quark model wave function}

A light-cone quark model wave function for the pion was given by Dziembowski and Mankiewicz (DM) ${ }^{15}$ and it has received attention for the reason that it can fit the static properties, the form factor, and the $\mathrm{CZ}$ distribution amplitude for the pion simultaneously. We indicate that this wave function, though is good in "shape", has serious problems in "magnitude".

The main idea in Ref.15 is reasonable: When one transforms from equal-time (instant-form) wave function to light-cone wave function, one should consider, besides the momentum space wave function transformation such as the Brodsky-Huang-Lepage prescription, also the Melosh transformation relating equal-time spin wave functions and light-cone spin wave functions. The following assumptions and approximations were $\operatorname{made}^{15}$ :

1. It was assumed that the ground-state in the pion is described by the harmonic oscillator wave function, and adopted the Brodsky- Huang-Lepage prescription of the momentum space wave function.

2. They made a mock-meson assumption that mesons are a collection of quarks with all binding turned off and a mock-meson mass equals to the mean total energy of the free quarks.

3. They adopted the mock-meson mass $M_{M}=m_{\pi} / 4+3 m_{\rho} / 4=612 \mathrm{MeV}$ rather than the real mass $m_{\pi}=139 \mathrm{MeV}$ or the mean total energy of the two quarks $M_{M}=2<\left(m^{2}+\vec{k}^{2}\right)^{1 / 2}>>2\left(m^{2}+<\vec{k}_{\perp}^{2}>\right)^{1 / 2}=950 \mathrm{MeV}$ for the pion $\left(\right.$ with $\left.<\vec{k}_{\perp}^{2}>\approx(345 \mathrm{MeV})^{2}\right)$.

4. An approximation $k^{0}+m \approx 2 m$ was made in the obtained light-cone wave function, where $k^{0}=\left(m^{2}+\vec{k}^{2}\right)^{1 / 2}$.

5. It was impliedly assumed that the pion's helicity to be the sum of the light-cone helicity of quarks.

They got, upon the above assumptions and approximations, the $\lambda_{1}+\lambda_{2}=0$ com- 
ponent light-cone wave function for the pion,

$$
\psi\left(x, \vec{k}_{\perp}\right)=A \frac{a_{1} a_{2}-\vec{k}_{\perp}^{2}}{x(1-x)} \exp \left[-\frac{m^{2}+\vec{k}_{\perp}^{2}}{8 \beta^{2} x(1-x)}\right]
$$

where $a_{1}=x M_{M}+m$ and $a_{2}=(1-x) M_{M}+m$. From this wave function one obtains the distribution amplitude,

$$
\phi(x)=\frac{A}{16 \pi^{2} x_{1} x_{2}}\left[a_{1} a_{2}\left(8 \beta^{2} x_{1} x_{2}\right)-\left(8 \beta^{2} x_{1} x_{2}\right)^{2}\right] \exp \left[-\frac{m^{2}}{8 \beta^{2} x(1-x)}\right] .
$$

It was argued ${ }^{15}$ that the parameters $m=330 \mathrm{MeV}$ and $\beta=450 \mathrm{MeV}$ are reasonable at large momentum transfer. The distribution amplitude for these parameters is very close to the CZ-form, as can be seen from Fig.4. The parameter A should be fixed by the first constraint in Sec.II. Then one can find, from (3.15), the probability of finding the $q \bar{q}\left(\lambda_{1}+\lambda_{2}=0\right)$ Fock state in a pion,

$$
P_{q \bar{q}}=3.24
$$

which is unreasonablely large. If we require that $P_{q \bar{q}}$ be less than unity, then the magnitude of the calculated distribution amplitude will be less than $30 \%$ of that of the CZ distribution amplitude, and this distribution amplitude surely cannot fit the pion form factor data. The calculated structure function is,

$$
\begin{aligned}
F_{2}^{V}(x) & =\frac{5}{9} \frac{A^{2} x}{16 \pi^{2} x_{1}^{2} x_{2}^{2}}\left[2\left(4 \beta^{2} x_{1} x_{2}\right)^{3}-2 a_{1} a_{2}\left(4 \beta^{2} x_{1} x_{2}\right)^{2}\right. \\
& \left.+a_{1}^{2} a_{2}^{2}\left(4 \beta^{2} x_{1} x_{2}\right)\right] \exp \left[-\frac{m^{2}}{4 \beta^{2} x(1-x)}\right] .
\end{aligned}
$$

Fig.4(b) presents the comparison of the reduced (by a factor 0.1) valence state structure function with the NA3 parameterization of the pion structure function. It can be seen that the calculated $F_{2}^{V}$ is almost two orders of magnitude larger than the experimental structure function data as $x \rightarrow 1$. Thereby the pion wave function (3.15) though is good in reproducing the shapes of the $\mathrm{CZ}$ distribution amplitude and reasonable valence state structure function, it has serious problems in producing the correct magnitude.

The probability may be less than 1 if we change the parameters. For example, we find $P_{q \bar{q}} \approx 0.86$ in the case of $m=330 \mathrm{MeV}$ and $\beta=330 \mathrm{MeV}$, which were used by many authors in studying the low momentum properties for hadrons in the constituent quark model framework ${ }^{24}$. However, the calculated distribution amplitude, as shown in Fig.4(a), differs significantly from the CZ-like distribution amplitude. 


\section{The power-law wavefunction ${ }^{25}$}

It is interesting to look for a wave function that can satisfy the four constraints:

$$
\begin{aligned}
& \text { (1) } \int_{0}^{1} d x_{1} \phi\left(x_{i}\right)=\frac{f_{\pi}}{2 \sqrt{3}} \\
& \text { (2) } \int_{0}^{1} d x_{1} \psi\left(x_{i}, \vec{k}_{\perp}=0\right)=\frac{\sqrt{3}}{f_{\pi}} \\
& \text { (3) } P_{q \bar{q}}=\int d x_{1}\left[d^{2} k_{\perp}\right]\left|\psi\left(x_{i}, \vec{k}_{\perp}\right)\right|^{2}<1 \\
& \text { (4) } G_{\nu / \pi}^{(2)}=\int\left[d^{2} \vec{k}_{\perp}\right]\left|\psi\left(x_{i}, \vec{k}_{\perp}\right)\right|^{2}=\text { at or below data. }
\end{aligned}
$$

Further, we should examine the electromagnetic form factor $F_{\pi}\left(Q^{2}\right)$ and the average transverse momentum $<k_{\perp}^{2}>$.

Now let us consider the power-law form of the pionic wave function

$$
\psi\left(x, \vec{k}_{\perp}=N \tilde{\phi}(\xi)\left(\frac{\vec{k}_{\perp}^{2}}{x_{1} x_{2} \beta}+1\right)^{-L}\right.
$$

where $\mathrm{L}$ and $\beta$ are some constants, $\xi=x_{1}-x_{2}$, and the normalization

$$
N=(L-1) \frac{16 \pi^{2}}{\beta}
$$

is chosen so that

$$
\phi=x_{1} x_{2} \tilde{\phi}
$$

For definiteness, let $\phi$ is $\mathrm{CZ}$ distribution amplitude, i.e.

$$
\tilde{\phi}=5 \sqrt{3} f_{\pi}\left(x_{1}-x_{2}\right)^{2}
$$

Then from the constraints one can get the following results:

$$
\begin{aligned}
P_{q \bar{q}} & =\frac{9}{14} \frac{L-1}{2 L-1} \leq \frac{9}{28} \text { for } L \geq 1 \\
G_{\nu / \pi}^{(2)} & =\int \frac{d^{2} k_{\perp}}{16 \pi^{3}}\left|\psi\left(x, \vec{k}_{\perp}\right)\right|^{2}=45 \frac{L-1}{2 L-1} x(1-x)(2 x-1)^{4} \\
<\vec{k}_{\perp}^{2}>^{1 / 2} & =\sqrt{\frac{40}{27}} \pi f_{\pi} \simeq 356 \mathrm{MeV} \text { independent of } L
\end{aligned}
$$

In order to fit the structure function data at $x \rightarrow 1$, we have a constraint that $\mathrm{L}$ is below some number. For example, we can put $L=\frac{59}{58}$ since a simple valence quark distribution function like

$$
G_{\nu / \pi}=\frac{3}{4} x^{-1 / 2}(1-x)
$$


gives tolerable agreement with data for $x \rightarrow 1$.

The example tell us it is possible to find wave functions that do succeed and these are the type that should be used in any future calculation.

\section{The Revised Light-cone Quark Model Wave Func- tion}

One can easily find that the assumptions 2 and 3 in Ref.15 are in fact inconsistent. The unreasonable large $P_{q \bar{q}}$ for the wave function (3.15) should be an indication of some unreasonable assumptions and approximations made in Ref.15. Therefore we re-construct the light-cone quark model wave function for the pion based upon the Kondratyuk- Terent'ev work $^{18}$ on the relativistically invariant wave function of twoparticle system in light-front dynamics.

We start our discussion from the $\mathrm{SU}(6)$ instant-form (T) wave function for the pion in the rest frame $\left(\overrightarrow{q_{1}}+\overrightarrow{q_{2}}=0\right)$,

$$
\psi_{T}\left(\vec{q}^{2}\right)=A \exp \left(-\vec{q}^{2} / 2 \beta^{2}\right)\left(\chi_{1}^{\uparrow} \chi_{2}^{\downarrow}-\chi_{1}^{\downarrow} \chi_{2}^{\uparrow}\right) / \sqrt{2}
$$

in which $\chi_{i}^{\uparrow, \downarrow}$ is the two-component Pauli spinor and the two quarks have 4-momenta $q_{1}^{\mu}=\left(q^{0}, \vec{q}\right)$ and $q_{2}^{\mu}=\left(q^{0},-\vec{q}\right)$, with $q^{0}=\left(m^{2}+\vec{q}^{2}\right)^{1 / 2}$, respectively. The instant-form spin states $\mid J, s>_{T}$ and the front-form $(\mathrm{F})$ spin states $\mid J, \lambda>_{F}$ are related by a Wigner rotation $U^{J}$ (Ref.26),

$$
\left|J, \lambda>_{F}=\sum_{s} U_{s \lambda}^{J}\right| J, s>_{T}
$$

and this rotation is called Melosh rotation for spin-1/2 particles. One should transform both sides of (4.1) simultaneously to get the light-cone wave function for the pion. For the left side, i.e., the pion, the transformation is particularly simple since the Wigner rotations are reduced to unity. For the right side, i.e., two spin-1/2 quarks, each particle instant-form and front-form spin states are related by the Melosh transformation ${ }^{17-18}$,

$$
\begin{aligned}
& \chi^{\uparrow}(T)=w\left[\left(q^{+}+m\right) \chi^{\uparrow}(F)-q^{R} \chi^{\downarrow}(F)\right] ; \\
& \chi^{\downarrow}(T)=w\left[\left(q^{+}+m\right) \chi^{\downarrow}(F)+q^{L} \chi^{\uparrow}(F)\right],
\end{aligned}
$$


where $w=\left[2 q^{+}\left(q^{0}+m\right)\right]^{-1 / 2}, \quad q^{R, L}=q^{1} \pm i q^{2}$, and $q^{+}=q^{0}+q^{3}$. We also adopt the Brodsky- Huang-Lepage prescription for the harmonic oscillator momentum space wave function transformation. Then we get the light-cone (or front-form) wave function for the pion,

$$
\psi\left(x, \vec{k}_{\perp}\right)=A \exp \left[-\frac{m^{2}+\vec{k}_{\perp}^{2}}{8 \beta^{2} x(1-x)}\right] \sum_{\lambda_{1}, \lambda_{2}} C_{0}^{F}\left(x, \vec{k}_{\perp}, \lambda_{1}, \lambda_{2}\right) \chi_{1}^{\lambda_{1}}(F) \chi_{2}^{\lambda_{2}}(F)
$$

where the component coefficients $C_{0}^{F}\left(x, \vec{k}_{\perp}, \lambda_{1}, \lambda_{2}\right)$ for $\mathrm{J}=1$, when expressed in terms of the instant-form momentum $q^{\mu}=\left(q^{0}, \vec{q}\right)$, have the forms,

$$
\begin{aligned}
& C_{0}^{F}\left(x, \vec{k}_{\perp}, \uparrow, \downarrow\right)=w_{1} w_{2}\left[\left(q_{1}^{+}+m\right)\left(q_{2}^{+}+m\right)-\vec{q}_{\perp}^{2}\right] / \sqrt{2} ; \\
& C_{0}^{F}\left(x, \vec{k}_{\perp}, \downarrow, \uparrow\right)=-w_{1} w_{2}\left[\left(q_{1}^{+}+m\right)\left(q_{2}^{+}+m\right)-\vec{q}_{\perp}^{2}\right] / \sqrt{2} ; \\
& C_{0}^{F}\left(x, \vec{k}_{\perp}, \uparrow, \uparrow\right)=w_{1} w_{2}\left[\left(q_{1}^{+}+m\right) q_{2}^{L}-\left(q_{2}^{+}+m\right) q_{1}^{L}\right] / \sqrt{2} ; \\
& C_{0}^{F}\left(x, \vec{k}_{\perp}, \downarrow, \downarrow\right)=w_{1} w_{2}\left[\left(q_{1}^{+}+m\right) q_{2}^{R}-\left(q_{2}^{+}+m\right) q_{1}^{R}\right] / \sqrt{2} ;
\end{aligned}
$$

which satisfy the relation,

$$
\sum_{\lambda_{1}, \lambda_{2}} C_{0}^{F}\left(x, \vec{k}_{\perp}, \lambda_{1}, \lambda_{2}\right)^{*} C_{0}^{F}\left(x, \vec{k}_{\perp}, \lambda_{1}, \lambda_{2}\right)=1
$$

It can be seen that there are two higher helicity $\left(\lambda_{1}+\lambda_{2}= \pm 1\right)$ components in the expression of the light- cone wave function for the pion besides the ordinary helicity $\left(\lambda_{1}+\lambda_{2}=0\right)$ components. These two higher helicity components arise from Wigner rotations (or Melosh rotations in a strict sense) ${ }^{27}$. We also indicate that one should express the instant-form momentum $\vec{q}=\left(q^{3}, \vec{q}_{\perp}\right)$ in terms of the light-cone momentum $\underline{k}=\left(x, \vec{k}_{\perp}\right)$ in the calculation. However, the relation between $\vec{q}$ and $\underline{k}$ is by no means unique, thus in practice one needs to construct models relating them. This leads us to discuss the following two possible schemes relating the instant-form momentum $\vec{q}=\left(q^{3}, \vec{q}_{\perp}\right)$ and the light-cone momentum $\underline{k}=\left(x, \vec{k}_{\perp}\right)$.

\section{a). Scheme one}

A reasonable connection between $\vec{q}$ and $\underline{k}$ was given in Ref.28-29,

$$
\begin{aligned}
x & =\left(q^{0}+q^{3}\right) / M ; \\
\vec{k}_{\perp} & =\vec{q}_{\perp},
\end{aligned}
$$

in which $\mathrm{M}$ satisfies

$$
M^{2}=\frac{\vec{k}_{\perp}^{2}+m^{2}}{x(1-x)}
$$


From Eq.(4.7) we find,

$$
\begin{aligned}
& q^{0}=\left[x M+\left(m^{2}+\vec{k}_{\perp}^{2}\right) / x M\right] / 2 \\
& q^{3}=\left[x M-\left(m^{2}+\vec{k}_{\perp}^{2}\right) / x M\right] / 2
\end{aligned}
$$

thus

$$
\begin{aligned}
& q^{+}=x M, \\
& 2 q^{+}\left(q^{0}+m\right)=(x M+m)^{2}+\vec{k}_{\perp}^{2} .
\end{aligned}
$$

We notice

$$
\vec{q}^{2}=\frac{\vec{k}_{\perp}^{2}+m^{2}}{4 x(1-x)}-m^{2}
$$

which is consistent with Eq.(3.2) in the Brodsky-Huang-lepage prescription ${ }^{3}$. The detailed reasons for the connection (4.7) can be found in Refs.28-29. Thus the $\lambda_{1}+\lambda_{2}=$ 0 component wave function can be obtained,

$$
\psi\left(x, \vec{k}_{\perp}\right)=A \frac{a_{1} a_{2}-\vec{k}_{\perp}^{2}}{\left[\left(a_{1}^{2}+\vec{k}_{\perp}^{2}\right)\left(a_{2}^{2}+\vec{k}_{\perp}^{2}\right)\right]^{1 / 2}} \exp \left[-\frac{m^{2}+\vec{k}_{\perp}^{2}}{8 \beta^{2} x(1-x)}\right]
$$

where $a_{1}=x M+m$ and $a_{2}=(1-x) M+m$. The constraint $P_{q \bar{q}}<1$ will be satisfied with any reasonable $\mathrm{m}$ and $\beta$ by using the first constraint to fix the parameter $\mathrm{A}$. Fig.5 presents the calculated distribution amplitudes and structure functions with two sets of parameters, i.e., $m=330 \mathrm{MeV}, \quad \beta=540 \mathrm{MeV}$, and $m=\beta=330 \mathrm{MeV}$, respectively, for the wave function (4.12). When compared with the results from the BHL wave function (3.15) in Fig.1, we find, in contrary to the claims in Ref.15, that the effect from Melosh rotation seems to have no significant effect on both the calculated distribution amplitude and the valence state structure function. The corresponding $P_{q \bar{q}}$ for the two set parameters are 0.228 and 0.552 , respectively.

\section{b). Scheme two}

We adopt the assumptions 1 and 2 in Sec.III as the starting point for scheme two. In this case the relation between $\vec{q}$ and $\underline{k}$ is very simple,

$$
\begin{aligned}
& x=\left(q^{0}+q^{3}\right) / M \\
& \vec{k}_{\perp}=\vec{q}_{\perp}
\end{aligned}
$$

where $\mathrm{M}$, the mock-meson mass, defined to be the mean total energy of the free quarks,

$$
M=2<\left(m^{2}+\vec{q}^{2}\right)^{1 / 2}>\approx 2\left(m^{2}+3 / 2<\vec{k}_{\perp}^{2}>\right)^{1 / 2}
$$


the value of which should be, approximately, $1130 \mathrm{MeV}$ and $910 \mathrm{MeV}$ for the above two sets of parameters with $\left\langle\vec{k}_{\perp}^{2}>\right.$ being $(374 \mathrm{MeV})^{2}$ and $(256 \mathrm{MeV})^{2}$ respectively in scheme one. From Eq.(4.13) it follows,

$$
\begin{aligned}
& q^{+}=x M ; \\
& 2 q^{+}\left(q^{0}+m\right)=(x M+m)^{2}+\vec{k}_{\perp}^{2},
\end{aligned}
$$

Thereby the $\lambda_{1}+\lambda_{2}=0$ component wave function should be (4.12) with fixed M rather than $\mathrm{M}$ in Eq.(4.8). We find $P_{q \bar{q}}$ to be 0.484 and 0.723 respectively, which are larger than those in scheme one. The calculated distribution amplitudes and structure functions, as presented in Fig.6, are also larger than those in scheme one. We also calculate the distribution amplitude and the valence state structure function for the first set parameters by using $\mathrm{M}=612 \mathrm{MeV}$ and compared them with the results in Ref.15 as shown in Fig.4. It can be seen that the distribution amplitude is close to the $\mathrm{CZ}$-form, with also the unreasonablely large valence state structure function as that in Sec.III.C. The probability $P_{q \bar{q}}$ is 2.93 , which is larger than unity. Thus the flaws suffered by the wave function (3.15) mainly raised from the inconsistent assumption 3; i.e., the use of a smaller M. The approximation 4 also has a large consequence on the results, thereby this approximation seems to be too strong. We also notice that

$$
\vec{q}^{2}=\frac{1}{4}\left[x M+\left(\frac{m^{2}+\vec{k}_{\perp}^{2}}{x M}\right)\right]^{2}-m^{2},
$$

which is inconsistent with Eq.(3.2). Further observation of the unreasonableness of the results in Ref.15 is that the effect from Melosh rotation should disappear for $\vec{k}_{\perp}=0$. This aspects is satisfied for the wave function (4.12), whereas it is not satisfied for the wave function (3.15).

We only comment without argument here that there are ambiguities of introducing a factor, such as $\sqrt{1 / 2 x_{1} x_{2}}$ adopted in Ref.28 or $\sqrt{M / 4 x_{1} x_{2}}$ adopted in Ref.29, to the Brodsky-Huang-Lepage wave function as a consequence from the jacobian relating instant-form momentum and light-cone momentum. However, the qualitative conclusions in above analyses will not changed though the quantitative results will be different if the factor is introduced. 


\section{Summary and Comments}

After the analysis of several existing pion wave functions in light-cone formalism and the re-construction of the light-cone quark model wave function, it is shown that the present wave functions $\left(\lambda_{1}+\lambda_{2}=0\right.$ component) have failed the requirements to fit the pion form factor data and the reasonable valence Fock state structure function which does not exceed the pion structure function data with reasonable parameters. However, as an example, we consider a power-law form of the pionic wave function. It is shown that all of constraints can be satisfied as $L=\frac{59}{58}$. This means that it is possible to find wave functions that do succeed and these are the type that should be used in any future calculation.

We also find, in contrary to pervious claims, that the effect from Melosh rotation in the ordinary helicity $\left(\lambda_{1}+\lambda_{2}=0\right)$ component wave function cannot reproduce a CZlike distribution amplitude. However, as mentioned in Sec.I, it is still an open problem whether the CZ-like distribution amplitude is the correct pion distribution amplitude, and the large second moment of the $\mathrm{CZ}$ distribution amplitude are not reproduced by a recent improved lattice QCD calculation. Thus we could consider the quark model evaluation of this paper as a suggestion that the pion distribution amplitude may close to the asymptotic form rather than to the $\mathrm{CZ}$ form.

Then we meet the problem that the "naive" asymptotic pion distribution amplitude can only account for about $50 \%$ of the existing pion form factor data. In previous perturbative QCD work the adoption of a CZ-like distribution amplitude was found to be a possible way to resolve this problem. We speculate in this paper that the introduction of the higher helicity states may provide an alternative way to address the problem concerning the applicability of perturbative QCD. These higher helicity components emerge naturally in the full light-cone wave function as a consequence from the Wigner rotation ${ }^{26}$ relating spin states in different frames. The existence of the higher helicity components in the light-cone wave function for the pion is not in contradiction with the requirement of angular momentum conservation, since the full light-cone wave function, e.g., (4.4), is an eigenstate of the total spin operator $I^{2}$ in front-form dynamics ${ }^{29}$. 
The introduction of the higher helicity component wave functions into the lightcone formalism may have significant consequence in several problems concerning the applicability of perturbative QCD. It is speculated that the perturbative contributions from the higher helicity states may provide the other fraction needed to fit the pion form factor data besides the perturbative contributions from the ordinary helicity states evaluated by using the "naive" Brodsky-Huang-Lepage distribution amplitude. However, there are still some difficulties in incorporating the higher helicity component wave functions into the conventional perturbative QCD framework since the distribution amplitudes for these components vanish from Eq.(2.5). Thereby it is necessary to developed a technique which enables us to calculate the perturbative contributions from these components. Some progress has been made in this direction and it will be given elsewhere. 


\section{References}

[1] See, e.g., S. J. Brodsky and G. P. Lepage, in Perturbative Quantum Chromodynamics, edited by A. H. Mueller (World Scientific, Singapore, 1989); and references therein.

[2] G. P. Lepage and S. J. Brodsky, Phys. Rev. D22, 2157(1980); S. J. Brodsky, Y. Frishman, G. P. Lepage, and C. Sachrajda, Phys. Lett. 91B, 239(1980).

[3] S. J. Brodsky, T. Huang, and G. P. lepage, in Particles and Fields, edited by A. Z. Capri and A. N. Kamal (Plenum Publishing Corporation, New York, 1983), p.143; G. P. Lepage, S. J. Brodsky, T. Huang and P. B. Mackenzie, ibid, p.83; T. Huang, in Proceedings of XX-th International Conference on High Energy Physics, Madison, Wisconsin, July 17-23, 1980, edited by L. Durand and L. G. Pondrom (American Institute of Physics, New York, 1981), p.1000.

[4] V. L. Chernyak and A. R. Zhitnitsky, Nucl. Phys. B201, 492(1982); Phys. Rep. 112, 173(1984); Nucl. Phys. B246, 52(1984).

[5] T. Huang, X. D. Xiang, and X. N. Wang, Chinese Phys. Lett. 2, 76(1985); Commun. Theor. Phys. 5, 117(1986); Phys. Rev. D35, 1013(1987).

[6] S. Gottlieb and A. S. Kronfeld, Phys. Rev. Lett. 55, 2531(1985); Phys. Rev. D33, 227(1986); G. Martinelli and C. T. Sachrajda, Phys. Lett. 217B, 319(1989).

[7] D. Daniel, R. Gupta, and D. G. Richards, Phys. Rev. D43, 3715(1991).

[8] E. E. Salpeter and H. A. Bethe, Phys. Rev. 84, 1232(1951).

[9] H. C. Pauli and S. J. Brodsky, Phys. Rev. D32, 1993, 2001(1985); T. Eller, H. C. Pauli, and S. J. Brodsky, Phys. Rev. D35, 1493(1987). See, also, A. C. Tang, SLAC-Report-351(1990), and references therein.

[10] S. J. Brodsky, C.-R. Ji, and M. Sawicki, Phys. Rev. D32, 1530(1985); T. Huang and Z. Huang, Commun. Theor. Phys. 11, 479(1989). 
[11] O. C. Jacob and L. S. Kisslinger, Phys. Lett. 243B, 323(1990); L. S. Kisslinger and O. C. Jacob, in Nuclear and Particle Physics on the Light Cone, edited by M. B. Johnson and L. S. Kisslinger (World Scientific, Singapore, 1989), p.322.

[12] T. Huang, in Proceedings of the International Symposium on Particle and Nuclear Physics, Beijing, Sept. 2-7, 1985, edited by N. Hu and C. S. Wu, (World Scientific, Singapore, 1986), p.151; and in Proceedings of Socond Asia Pacific Physics Conference, India, 1986, (World Scientific, Singapore, 1987), p.258.

[13] T. Huang, Nucl. Phys. B(Proc. Suppl.)7B, 320(1989); T. Huang and Q. X. Shen, Z. Phys. C50, 139(1991).

[14] C. E. Carson and F. Gross, Phys. Rev. D36, 2060(1987).

[15] Z. Dziembowski and L. Mankiewicz, Phys. Rev. Lett. 58, 2175(1987); Z. Dziembowski, Phys. Rev. D37, 778(1987); and in Nuclear and Particle Physics on the Light Cone, edited by M. B. Johnson and L. S. Kisslinger (World Scientific, Singapore, 1989), p.166.

[16] See, e.g., W. J. Metcalf et al., Phys. Lett. 91B, 275(1980).

[17] H. J. Melosh, Phys. Rev. D9, 1095(1974).

[18] L. A. Kondratyuk and M. V. Terent'ev, Yad. Fiz. 31, 1087(1980) [Sov. J. Nucl. Phys. 31, 561(1980)].

[19] J. Badier et al., Z. Phys. C18, 281(1983).

[20] A. Altarelli and G. Parisi, Nucl. Phys. B126, 298(1977).

[21] B. Q. Ma, Phys. Lett. 176B, 179(1986); B.Q. Ma and J. Sun, Int. J. Mod. Phys. A6, 345(1991).

[22] See, e.g., Elementary Particle Theory Group, Peking University, Acta Physics Sinica 25, 415(1976); N. Isgur, in The New Aspects of Subnuclear Physics, edited by A. Zichichi (Plenum, New York, 1980), p.107.

[23] V. M. Braun and I. E. Filyanov, Z. Phys. C44, 157(1989). 
[24] H. J. Weber, Ann. Phys. (N. Y.)177, 38(1987); K. Konen and H. J. Weber, Phys. Rev. D41, 2201(1990), and references therein.

[25] Thanks referee for making a comment on the power-law wave function.

[26] E. Wigner, Ann. Math. 40, 149(1939).

[27] N. Isgur and C. H. Llewellyn Smith, Nucl. Phys. B317, 526(1989).

[28] V. A. Karmanov, Nucl. Phys. B166, 378(1980); ibid. A362, 331(1981).

[29] F. Coester, in Constraint's Theory and Relativistic Dynamics, edited by G. Longhi and L. Lusanna (World Scientific, Singapore, 1987), p.159; and in The Three-Body Force in the Three-Nucleon System, edited by B. L. Berman and B. F. Gibson (Springer, New York, 1986), p.472. 


\section{Figure Captions}

Fig.1 (a). The normalized distribution amplitude $\hat{\phi}(x)=\phi(x) / \sqrt{3} f_{\pi}$ : curves CZ and AS are the Chernyak-Zhitnitsky distribution amplitude (Ref.4) and the asymptonic distribution amplitude (Ref.3); curve BHL is the distribution amplitude from the Brodsky-Huang-Lepage wave function (3.5) (Ref.3), respectively. (b). The structure function: curve NA3 is the NA3 parameterization of the pion structure function; curve BHL is the valence state structure function for the Brodsky-Huang-Lepage wave function.

Fig.2 Similar as in Fig.1. (a). Curve $H$ is the distribution amplitude (3.11) from Huang's factorized ansatz (Refs.12-13). (b). Curve $\mathrm{H}$ is the valence state structure function for Huang's factorized wave function.

Fig.3 Similar as in Fig.1. Curves BF(I) and BF(II) are the results from the factorized wave functions, with $S\left(x_{1}-x_{2}\right)$ to be the two sets Braun-Filyanov (Ref.24) like distribution amplitudes, for the parameters $m=324 \mathrm{MeV}, \beta=432 \mathrm{MeV}$, and $m=346 \mathrm{MeV}, \quad \beta=461 \mathrm{MeV}$, respectively.

Fig.4 Similar as in Fig.1. Curves DM(1) and DM(2) are the results from the DziembowskiMankiewicz (Ref.15) wave function (3.15) with the parameters to be $m=330 \mathrm{MeV}, \beta=$ $450 \mathrm{MeV}$, and $m=330 \mathrm{MeV}, \beta=330 \mathrm{MeV}$, respectively.

Fig.5 Similar as in Fig.1. Curves a and b are the results from the $\left(\lambda_{1}+\lambda_{2}=0\right)$ component light-cone wave function (4.12), with $\mathrm{M}$ in Eq.(4.8), for scheme one with the parameters to be $m=330 \mathrm{MeV}, \quad \beta=540 \mathrm{MeV}$, and $m=330 \mathrm{MeV}, \beta=$ $330 \mathrm{MeV}$, respectively.

Fig.6 Similar as in Fig.1. Curves a, b and c are the results from the $\left(\lambda_{1}+\lambda_{2}=0\right)$ component light-cone wave function (4.12), with fixed M, for scheme two with the parameters to be: $m=330 \mathrm{MeV}, \quad M=1130 \mathrm{MeV}$ and $\beta=540 \mathrm{MeV} ; m=$ $330 \mathrm{MeV}, \quad M=910 \mathrm{MeV}$ and $\beta=330 \mathrm{MeV}$; and $m=330 \mathrm{MeV}, \mathrm{M}=612 \mathrm{MeV}$ and $\beta=540 \mathrm{MeV}$. 